\title{
Transformation of Phase Size Distribution into Scattering Intensity
}

\author{
BARRY J. BAUER \\ Polymers Division, Materials Science and Engineering Laboratory, National Institute of Standards and Technology, \\ 100 Bureau Drive, Mail Stop 8541, Gaithersburg, Maryland 20899-8541
}

Received 28 August 2003; revised 15 January 2004; accepted 20 January 2004

DOI: 10.1002/polb.20174

Published online in Wiley InterScience (www.interscience.wiley.com).

\begin{abstract}
A practical method of calculating the small-angle scattering intensity and the density correlation function from the phase size distribution is presented for a sample with a random two-phase morphology. The correlation function can be calculated in terms of joint probability distribution functions of the phase size distributions of the two individual phases with information from the chord length distribution. The phase size distribution is approximated as a weighted sum of exponentials, which is then transformed analytically into the correlation function and hence the small-angle scattering for any combination of phase size distributions of the two phases. This represents an extension of the Debye method for materials with more complex phase size distributions. The inverse problem of calculating the phase size distributions from the small-angle scattering requires a thermodynamic model or simplifying approximation. An example of the reverse transformation is given for a nanoporous polymer thin film. () 2004 Wiley Periodicals, Inc.* J Polym Sci Part B: Polym Phys 42: 3070-3080, 2004

Keywords: chord length; high concentration; pores; scattering; two-phase; neutron scattering; SAXS; phase separation
\end{abstract}

\section{INTRODUCTION}

Small-angle scattering has been widely used to measure characteristic sizes of an isotropic twophase system for phases with uniform internal scattering density (and, therefore, no contribution to the scattering from the internal structure of either phase) and sharp interfaces between the phases (and thus, no contribution to the scattering from the interfacial structure). ${ }^{1}$ Recently, this technique has been used to measure average pore sizes and distributions in nanoporous thin films

Official contribution of the National Institute of Standards and Technology; not subject to copyright in the United States.

Correspondence to: B. J. Bauer (E-mail: barry.bauer@ nist.gov)

Journal of Polymer Science: Part B: Polymer Physics, Vol. 42, 3070-3080 (2004) (c) 2004 Wiley Periodicals, Inc. *This article is a US Government work and, as such, is in the public domain in the United States of America made from polymeric precursors. ${ }^{2-4}$ Although certain methods exist for specific morphologies such as polydisperse spheres embedded in a continuous matrix, ${ }^{3,5-8}$ no general fitting method is available for random, nonspherical morphologies.

Porod $^{1,9}$ contributed two basic principles to two-phase scattering: the scattering invariant and the high q scattering limit, which has been termed Porod's law:

$$
Q=\frac{K_{n}}{2 \pi^{2}} \int_{0}^{\infty} \mathbf{q}^{2} I(\mathbf{q}) d \mathbf{q}=V\left(\rho_{\mathrm{A}}-\rho_{\mathrm{B}}\right)^{2} \phi_{\mathrm{A}} \phi_{\mathrm{B}}
$$

The invariant $Q$ is the total scattered intensity $[I(\mathbf{q})]$ integrated over all values of the scattering vector $[\mathbf{q}=4 \pi \sin (\theta / 2) / \lambda$, where $\theta$ is the scattering angle and $\lambda$ is the wavelength]. $Q$ is a measure of 
the scattering volume $(V)$, the scattering contrast of phases $\mathrm{A}$ and $\mathrm{B}\left(\rho_{\mathrm{A}}\right.$ and $\left.\rho_{\mathrm{B}}\right)$, the volume fractions of phases $\mathrm{A}$ and $\mathrm{B},\left(\phi_{\mathrm{A}}\right.$ and $\left.\phi_{\mathrm{B}}\right)$, and the scattering contrast factor $\left(K_{n}\right)$ based on the composition of the phases.

Porod's law ${ }^{1,9}$ gives the limit at high q values:

$$
\mathbf{q}^{4} I(\mathbf{q} \rightarrow \infty)=2 \pi\left(\rho_{\mathrm{A}}-\rho_{\mathrm{B}}\right)^{2} S
$$

where $S$ is the total surface area of the interfaces. In eqs 1 and 2, contrast factors are used that do not normalize the scattered intensity to the scattering volume. Two important results of these equations are the high $\mathbf{q}$ power law of scattering from an ideal two-phase system of $\mathbf{q}^{-4}$ and the capability of calculating characteristic phase sizes from small-angle scattering. The ratios of the calculations in eqs 1 and 2 give the $V / S$ ratio, which gives the average chord length of phase $i$ $\left(l_{i}\right)$ as follows:

$$
l_{i}=4 \frac{V}{S} \phi_{i}
$$

Small-angle scattering, therefore, can be used to measure the number-average phase size for a two-phase morphology with any size distributions of the individual phases. This relationship can be used to determine the sizes of phases from scattering but contains no information on the nature of the size distributions present.

Debye et al. ${ }^{1,10}$ showed that the scattering from a dense two-phase material is related to the density pair correlation function through a Fourier transform:

$I(\mathbf{q})=4 \pi\left(\rho_{\mathrm{A}}-\rho_{\mathrm{B}}\right)^{2} \phi_{\mathrm{A}} \phi_{\mathrm{B}} \int_{0}^{\infty} R^{2} \gamma(R) \frac{\sin (\mathbf{q} R)}{\mathbf{q} R} \mathbf{d} R$

where $\gamma(R)$ is the correlation function defined by

$$
\gamma(R)=\int_{x} \eta(x) \eta(x+R) / \int_{x} \eta(x)^{2}
$$

and the function $\eta(x)$ is the scattering contrast at position $x$. The integration is taken over all space, giving the correlated density at distance $R$. Debye et al. examined a two-phase system, with every point located in the sample being either pure A or pure B.

Scattering from a two-phase system made up of spherical objects dispersed in a continuous phase of a contrasting density has been calculated with a Percus-Yevick formalism. ${ }^{5-8}$ The spheres can be monodisperse or polydisperse and are ordered by energy constraints. The simplest constraint is nonoverlap of the spheres, ${ }^{6,7}$ but additional constraints of ionic interactions or excluded sphere radius have been added. ${ }^{8}$ Although such models are highly appropriate for morphologies that truly have a spherical phase, such as preformed spheres dispersed in a medium, ${ }^{3}$ they are not appropriate for morphologies that have cocontinuous phases or extremely broad size distributions. The fits are for model distributions, such as a Shultz distribution or a lognormal distribution. More complicated distribution types such as bimodal distributions are possible but have not been reported.

Two-phase morphologies that result from processes such as spinodal decomposition have also been described. ${ }^{11}$ The scattering from such systems can be used to measure structural parameters but is necessarily constrained by systems that obey the assumed thermodynamic conditions. Statistical descriptions for random morphologies have also been described. ${ }^{12,13}$ They are useful methods but require fitting statistical parameters that are often not intuitive.

Mering and Tchoubar ${ }^{14-17}$ used small-angle scattering to calculate the chord length distribution of samples with a two-phase morphology. ${ }^{18,19}$ The second derivative of the pair correlation function can be written in terms of the summation of joint phase size distributions. The joint probability distribution of series of alternating phases of type A and type B with $i$ total A phases and $j$ total B phases is designated $P_{\mathrm{A} i \mathrm{~B} j}$. Because of the alternation constraint, $i$ and $j$ can differ by no more than 1:

$$
\begin{aligned}
& \frac{\partial^{2} \gamma(R)}{\partial R^{2}}\langle l\rangle=\sum_{i=1}^{\infty} P_{\mathrm{A}_{i-1} \mathrm{~B}_{i}}(R)+P_{\mathrm{A}_{i} \mathrm{~B}_{i-1}}(R)-2 P_{\mathrm{A}_{i} \mathrm{~B}_{i}}(R) \\
& \quad=P_{\mathrm{A}}+P_{\mathrm{B}}-2 P_{\mathrm{AB}}+P_{\mathrm{ABA}}+P_{\mathrm{BAB}}-2 P_{\mathrm{ABAB}}+\cdots
\end{aligned}
$$

where $P_{\mathrm{A} 0 \mathrm{~B} 1}$ is equal to $P_{\mathrm{B}}(R)$ and $P_{\mathrm{A} 1 \mathrm{~B} 0}$ is equal to $P_{\mathrm{A}}(R)$. The others are defined by eq 7 . Equation 8 defines the characteristic scattering length $(\langle l\rangle)$ : 


$$
\begin{gathered}
P_{\mathrm{A}_{i+k} \mathrm{~B}_{j+l} l}(R)=\int_{0}^{R} P_{\mathrm{A}_{i} \mathrm{~B}_{j}}\left(R^{*}\right) P_{\mathrm{A}_{k} \mathrm{~B}_{l}}\left(R-R^{*}\right) d R^{*} \\
\langle l\rangle=\phi_{\mathrm{B}} \int_{0}^{\infty} R P_{\mathrm{A}}(R) d R=\phi_{\mathrm{A}} \int_{0}^{\infty} R P_{\mathrm{B}}(R) d R
\end{gathered}
$$

The nomenclature chord length distribution is somewhat misleading and should not be mistaken for a phase size distribution. Equation 6 demonstrates the difference between a phase size distribution and a chord length distribution. The individual terms $P_{\mathrm{A}}$ and $P_{\mathrm{B}}$ are the phase size distributions of phases $A$ and $B$, respectively. The entire right-hand side of eq 6 is called the chord length distribution and contains distribution functions of all possible multiple phase combinations. ${ }^{14-17}$ Although it is an important characteristic of the morphology, it is far less intuitive than the size distributions of the individual phases and has certain characteristics, such as negative values, which are, of course, impossible for the individual phase size distributions. Although the Mering-Tchoubar formalism describes the relationship between individual phase size distributions and the second derivative of the correlation function, no practical method has been described to convert an arbitrary phase size distribution into small-angle scattering and vice versa.

Smarsly and coworkers ${ }^{18-21}$ analyzed smallangle scattering with parameterization methods in which functional forms are assumed for the chord length distribution, which can be analytically transformed into small-angle scattering and fit to experimental results. This method advances the ability to extract information from experimental results but does not attempt to analyze the phase size distributions of the individual phases.

A Debye-type scatterer has an exponential correlation function, which results in a related exponential phase size distribution. ${ }^{10,14}$ Therefore, expressing a phase size distribution as a sum of exponentials is an appropriate form of data fitting. In this article, a procedure is introduced in which a phase size distribution can be fit with a weighted series of exponential functions of distance $R$. These functions can then be analytically transformed into a correlation function and hence small-angle scattering.

\section{THEORY}

The simplicity of eq 6 does not readily transfer into simplicity for the calculations necessary for finding an analytical solution for transformations of an arbitrary phase size distribution into a correlation function. Exact transformations for certain distributions are possible but are often cumbersome. It is possible, however, to use a truncated series of terms from eq 6 to calculate a correlation function from a phase size distribution. Truncations are sometimes made to include a single additional term in addition to the first term. ${ }^{14-17}$ An example is given of a model distribution to show how the number of terms used affects the calculation of the correlation function.

Because a Debye type of scatterer has an exponential phase size distribution, it is proposed that a sum of exponentials is an appropriate form of data fitting:

$$
P(R)=\sum_{i} w_{i} \exp \left(-R / R_{i}\right) / R_{i}
$$

The phase size distribution is approximated by a series of exponentials weighted by a factor $\left(w_{i}\right)$ having a characteristic length $\left(R_{i}\right)$. Each phase can be represented by any series of terms, with the phases having the same or different terms. For simplicity, subscripts A and B, denoting the two individual phases, are not shown in the righthand side of eq 9 .

Equation 7 can be used to calculate $P_{\mathrm{AB}}(R)$ from eq 9. If all values of $R_{i}$ are different, the result is given by eq 10 :

$$
\begin{aligned}
P_{\mathrm{AB}}(R)=\sum_{i} \sum_{j} w_{i} w_{j}( & \exp \left(-R / R_{i}\right) \\
& \left.-\exp \left[-R / R_{j}\right)\right] /\left(R_{i}-R_{j}\right)
\end{aligned}
$$

where $i$ and $j$ represent components $\mathrm{A}$ and $\mathrm{B}$, respectively. If $R_{i}$ is equal to $R_{j}$, then the integration gives

$$
P_{\mathrm{AB}}(R)=\sum_{i} w_{i}^{2} R \exp \left(-R / R_{i}\right)
$$

Further terms can be calculated with combinations of eqs 7,10 , and 11 . The exact functional form of the terms for joint distributions rapidly grows in complexity for larger numbers of phase combinations. The exact enumerations of the equation become lengthy after only a few terms.

However, combinations of exponentials with different $R_{i}$ and $R_{j}$ terms produce the same exponentials with multiplicative factors, which are functions of $w_{i}, w_{j}, R_{i}$, and $R_{j}$. When the $R_{i}$ and $R_{j}$ 


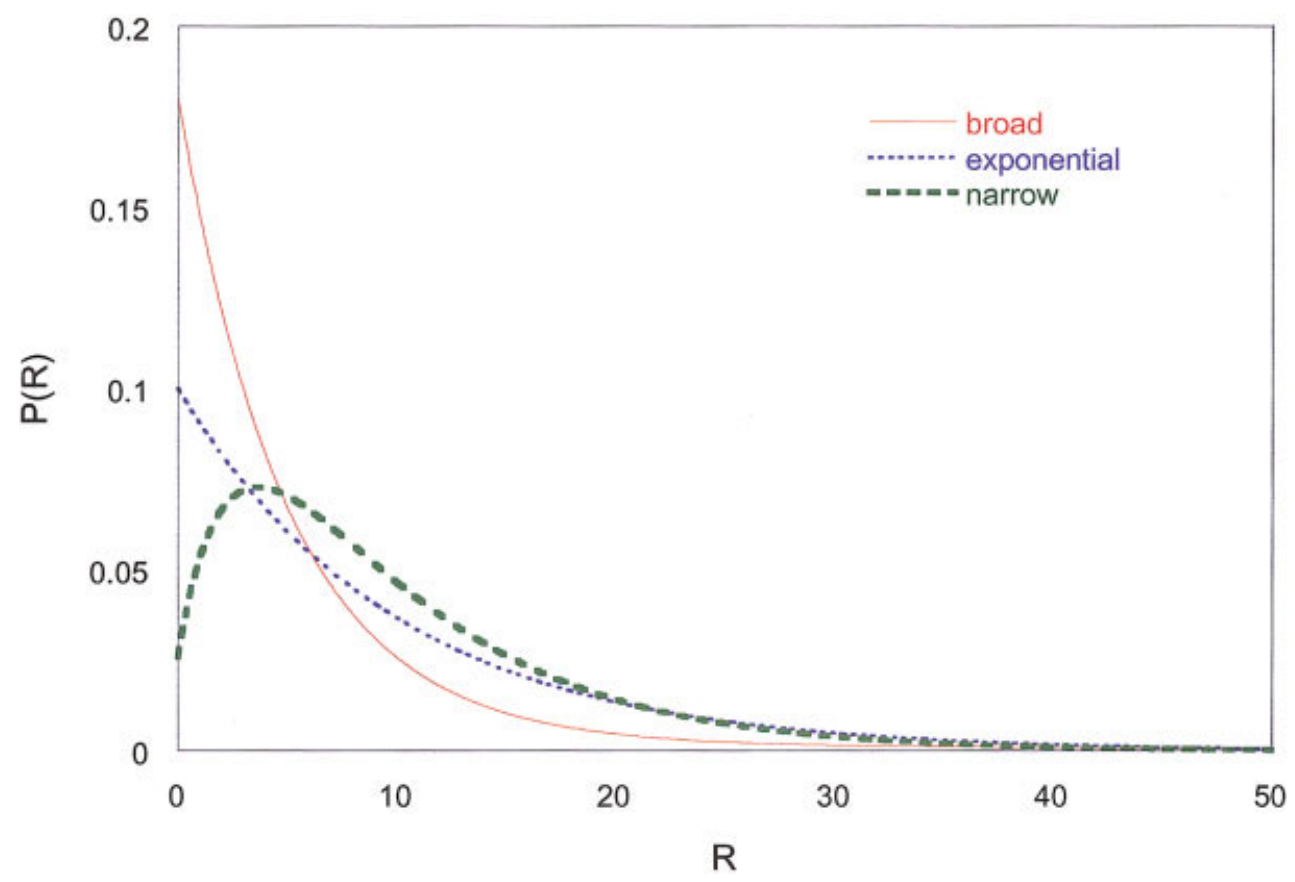

Figure 1. Phase size distributions of the model functions described by eqs 13-15. [Color figure can be viewed in the online issue, which is available at www.interscience.wiley.com]

terms are identical, the integration will result in exponentials with the same arguments but multiplied by polynomials in $R$, as demonstrated by eq 11. The rules for such a series of integrations are easily programmed, and tables can be kept of the multiplicative factors $\left(A_{m n}\right)$ in the following form:

$$
P_{\mathrm{A}_{k} \mathrm{~B}_{k}}(R)=\sum_{\text {all } m} \sum_{n=0}^{k-1} A_{n m} R^{n} \exp \left(-R / R_{m}\right)
$$

where subscript $k$ indicates the number of phases, $m$ indicates the exponential term of eq 9 , and $n$ indicates the power of $R$ in the polynomial.

As an example, three representative phase size distributions are considered: an exponential Debye type of distribution, a bimodal and broad exponential distribution, and a narrow distribution. The distribution parameters are shown in eqs 13-15, respectively:

$$
P_{\mathrm{A}}(R)=\exp (-R / 10) / 10 \quad \text { Exponential }
$$

$$
\begin{aligned}
& P_{\mathrm{A}}(R)=(8 / 9) \exp (-R / 5) / 5 \\
& +(1 / 9) \exp (-R / 50) / 50 \text { Broad }
\end{aligned}
$$

$$
\begin{gathered}
P_{\mathrm{A}}(R)=-0.012 \exp (-R / 1.0) / 1.0 \\
\quad-0.045 \exp (-R / 3.0) / 3.0 \\
+1.462 \exp (-R / 7.78) / 7.78 / 7.78 \quad \text { Narrow }
\end{gathered}
$$

In this example, the volume fraction of the $\mathrm{A}$ phase is $2 / 3$, and that of the $B$ phase is $1 / 3$. The values of the parameters were chosen to give identical number-average A phase sizes $\left(\int_{0}^{\infty} R P(R) d r / \int_{0}^{\infty} P(R) d r=10\right)$. The higher order average $\left(\int_{0}^{\infty} R^{2} P(R) d r / \int_{0}^{\infty} R P(R) d r\right)$ gives values of 20.0, 60.0, and 16.87 for the exponential, broad, and narrow distributions, respectively. The units of the distributions are arbitrary in these examples and are left off for clarity. Figure 1 is a plot of the three phase size distributions showing the three model distributions. The exponential (Debye) distribution is the random case, which is common for many two-phase systems; the other two examples are considerably narrower and broader than the random case. Equations 6 and 12 can be used to calculate the second derivative of the correlation function via the addition of the terms of the summation. It is also simple to integrate these terms twice to produce the correlation function itself. This form is more readily compared with the scattering results.

Figure 2 is a plot of the joint phase size distributions calculated from eqs 13-15. Joint probability dis- 


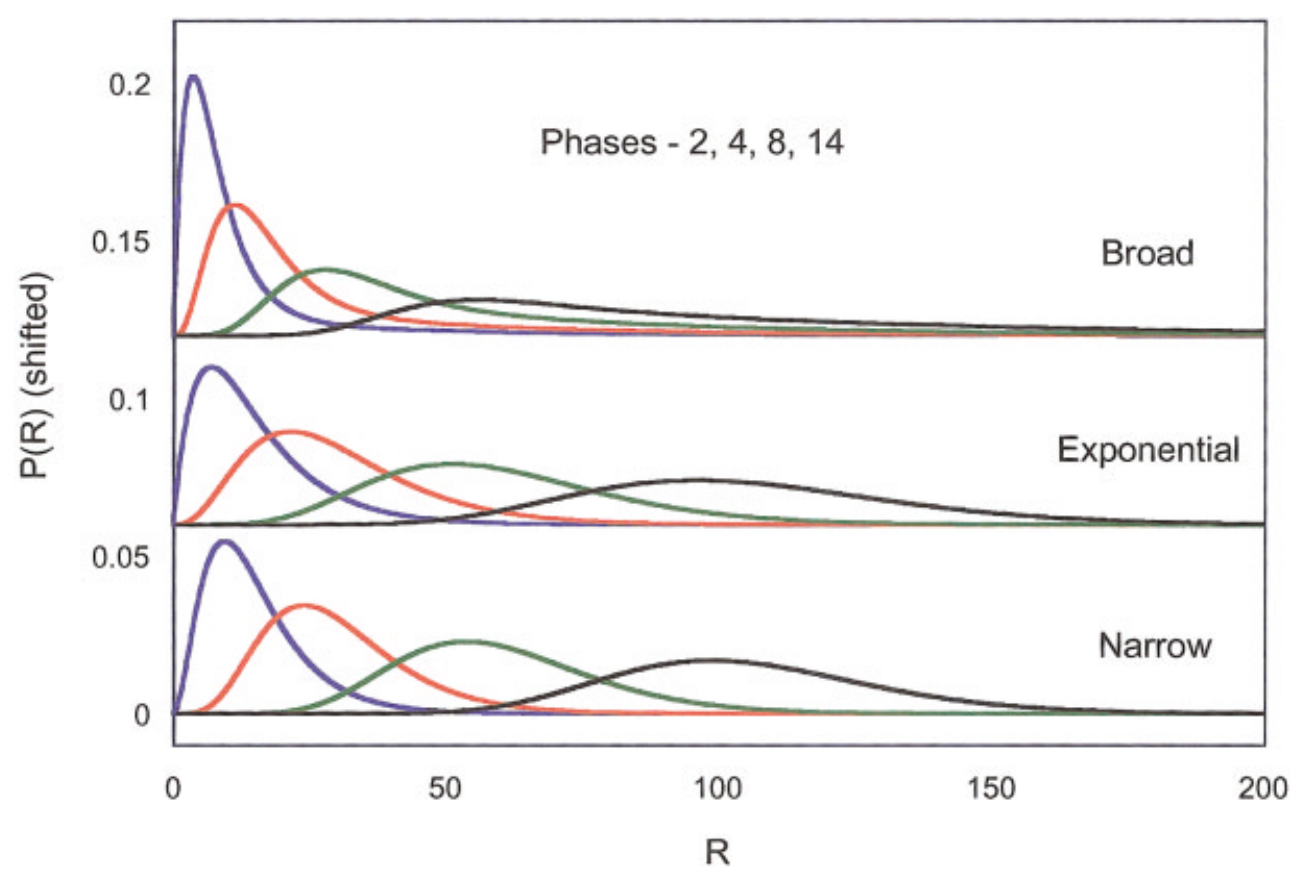

Figure 2. Joint phase size distributions of the model functions described by eqs 13-15. The curves from left to right show the joint phase size distribution of $A B, A_{2} B_{2}$, $\mathrm{A}_{4} \mathrm{~B}_{4}$, and $\mathrm{A}_{7} \mathrm{~B}_{7}$. [Color figure can be viewed in the online issue, which is available at www.interscience.wiley.com]

tributions are calculated up to a total of 14 consecutive phases in this figure. Figure 3 shows the correlation functions of the three model distributions based on truncated summations of eq 6 . For an even number of phases, the deviation due to the truncation is downward, but the point of deviation becomes much larger than the average phase size with a total number of phases that is easily calculated.

Converting this correlation function into smallangle scattering is not practical, however. The transformation of eq 4 requires a correlation function that goes to infinite correlation distances, and a transformation of a truncated sum of eq 6 results in anomalous oscillations in the scattering. In principle, a transformation of scattering data to the correlation function could be made with standard techniques, ${ }^{1}$ and these correlation function results could be compared with the results shown in Figure 3. Transformations of scattered intensity are always limited by the finite $\mathbf{q}$ range of the small-angle scattering data. Truncations of the scattering model used to fit the data are an additional source of uncertainty but can be eliminated by the use of transformation methods, which account for the complete set of terms in eq 6 without any truncation.

The results shown in Figure 3 also exemplify the difficulty in using truncated series from eq 6 to describe the scattering from dense two-phase systems, as previously described. ${ }^{14-17}$ Approximations of this sort are only valid for systems in which one phase is extremely dilute. A preferable method involves using the limit of an infinite summation of the probability terms of eq 6 , which is possible through transformation methods.

\section{LAPLACE TRANSFORMATION}

Mering and Tchoubar ${ }^{14-17}$ showed that transformation methods provide a practical method of calculating the correlation function from a phase size distribution in the limiting case of an infinite summation of all the factors in eq 6. If a Laplace transformation is taken of both sides of eq 6 , eq 16 results, $a(s)$ being the transform of $P_{\mathrm{A}}(R), b(s)$ being the transform of $P_{\mathrm{B}}(R)$, and $Q_{\gamma}(s)$ being the transform of $\delta^{2} \gamma / \delta R^{2}$ :

$$
Q_{\gamma}(s) \phi_{\mathrm{B}} \int_{0}^{\infty} R_{\mathrm{A}} P\left(R_{\mathrm{A}}\right) d R_{\mathrm{A}}=\frac{a(s)+b(s)-2 a(s) b(s)}{1-a(s) b(s)}
$$

Because the probability terms for phases A and B are sums of exponentials, as given by eq 9 , the $a(s)$ and $b(s)$ terms are also simple summations: 


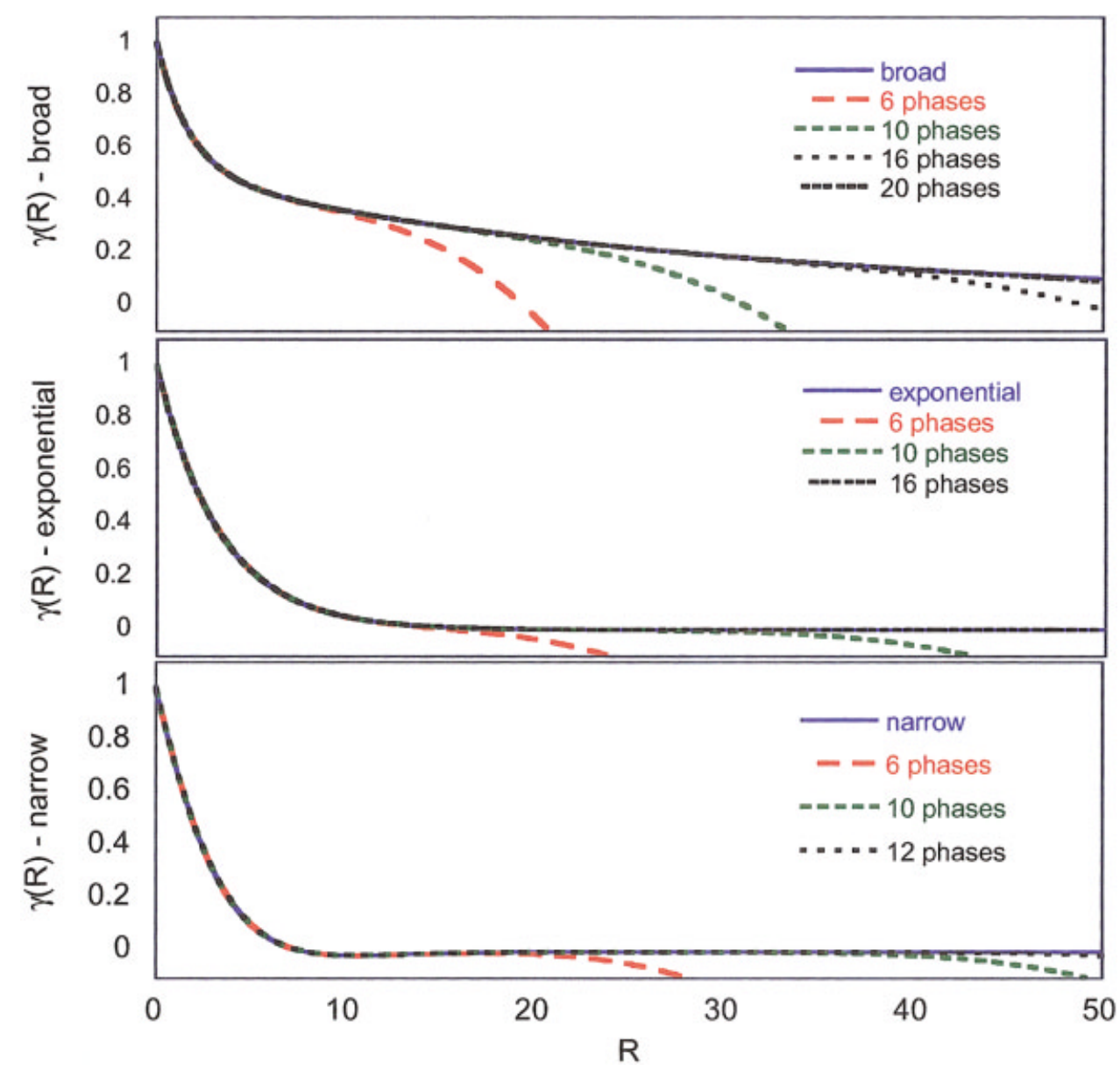

Figure 3. Truncated correlation functions of the model functions described by eqs 13-15 from sums of joint phase size distributions. [Color figure can be viewed in the online issue, which is available at www.interscience.wiley.com]

$$
a(s)=\sum_{i} \frac{w_{i} / R_{i}}{s+1 / R_{i}}
$$

All of the terms in eq 16 can be expanded into polynomials in $s$. The problem becomes equivalent to problems of signal analysis of electrical circuits of inductors, resistors, and capacitors. Finding the roots of the denominator and using partial fraction expansion generates a sum of terms that undergo a simple inverse transformation into three types, exponentials terms, exponential sine terms, and exponential cosine terms:

$$
\begin{aligned}
& \frac{\partial^{2} \gamma(R)}{\partial R^{2}}=\sum_{i} X_{i} \exp \left(-R / R_{i}\right) \\
& \quad+\sum_{j} Y_{j} \exp \left(-R / R_{j}\right) \sin \left(R / R_{j}\right) \\
& \quad+\sum_{k} Z_{k} \exp \left(-R / R_{k}\right) \cos \left(R / R_{k}\right)
\end{aligned}
$$

where the $X, Y, Z$, and $R$ terms are solutions to roots and expansion constants of the system. These terms can be easily integrated to yield the values of $\gamma(R)$, which has exactly the same functional form as eq 18.

Another advantage of assuming a fitting function of a sum of exponentials, as in eq 9 , is that they can easily be transformed into the scattered intensity through eq 4 . The transformations of the three types of terms are given by eqs 19-21:

$$
\begin{gathered}
\int_{0}^{\infty} R^{2} \exp \left(-R / R_{1}\right) \frac{\sin (\mathbf{q} R)}{\mathbf{q} R} d R=\frac{2 R_{1}^{3}}{\left(\beta^{2}+1\right)^{2}} \\
\int_{0}^{\infty} R^{2} \exp \left(-R / R_{1}\right) \sin \left(R / R_{2}\right) \frac{\sin (\mathbf{q} R)}{\mathbf{q} R} d R \\
=\frac{2 R_{1}^{3} \alpha\left[3-\alpha^{4}+2 \beta^{2}-\beta^{4}+2 \alpha^{2}\left(\beta^{2}+1\right)\right]}{\left[\alpha^{4}+2 \alpha^{2}\left(\beta^{2}-1\right)+\left(\beta^{2}+1\right)^{2}\right]^{2}}
\end{gathered}
$$




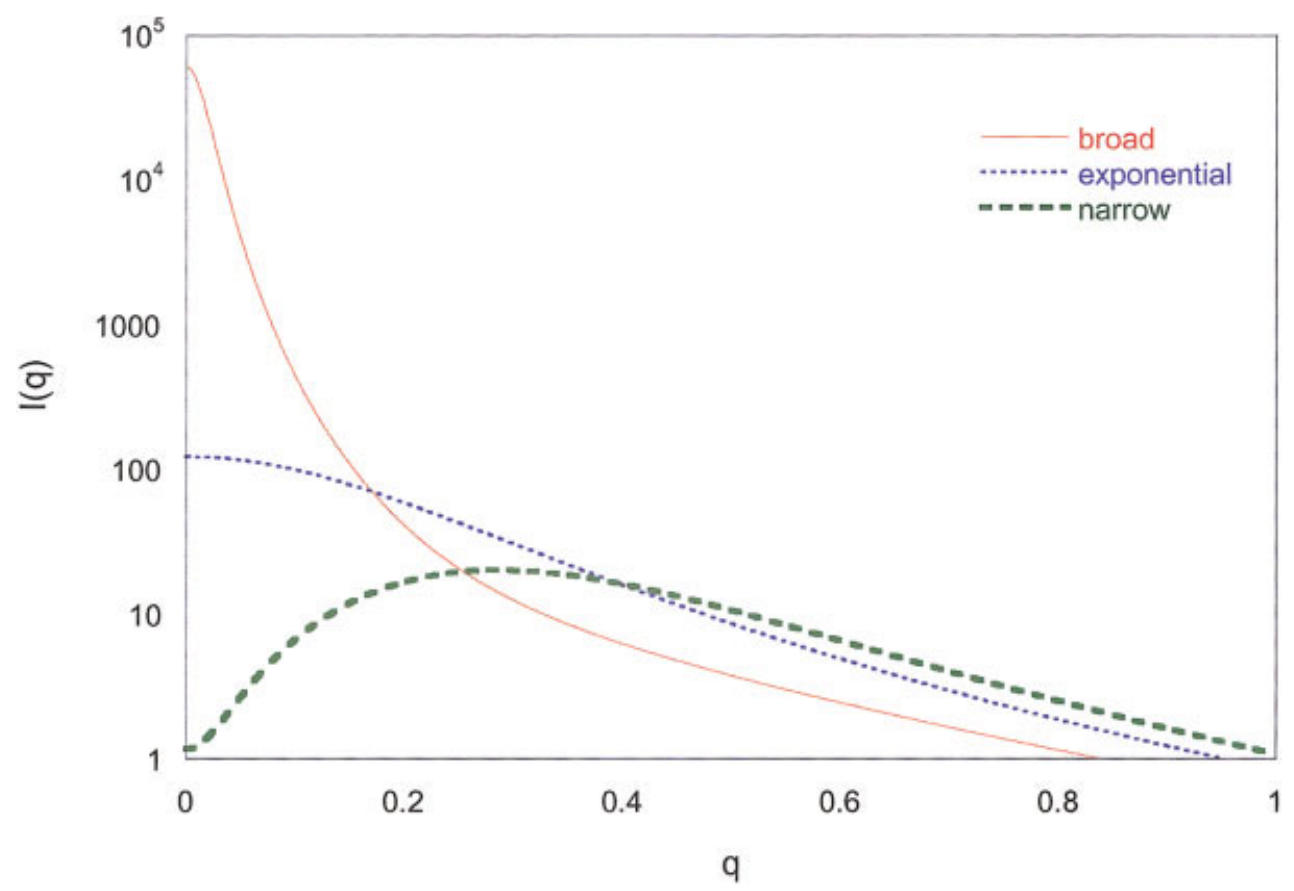

Figure 4. Scattering intensity of the model functions described in the text. [Color figure can be viewed in the online issue, which is available at www.interscience.wiley. com]

$$
\begin{aligned}
\int_{0}^{\infty} R^{2} \exp \left(-R / R_{1}\right) \cos \left(R / R_{2}\right) \frac{\sin (\mathbf{q} R)}{\mathbf{q} R} d R \\
=\frac{2 R_{1}^{3}\left[-3 \alpha^{4}+\left(\beta^{2}+1\right)^{2}-2 \alpha^{2}\left(\beta^{2}-1\right)\right]}{\left(\alpha^{4}+2 \alpha^{2}\left(\beta^{2}-1\right)+\left(\beta^{2}+1\right)^{2}\right)^{2}}
\end{aligned}
$$

where $\alpha$ is equal to $R_{1} / R_{2}$ and $\beta$ is equal to $\mathbf{q} R_{1}$.

Figure 4 is a plot of the calculated small-angle scattering from the three model distributions. The shapes and small-angle scattering intensities vary greatly between the samples. The average phase size is identical, however, as can be shown by eqs $1-3$. Figure 5 is a plot of $I(\mathbf{q}) \mathbf{q}^{2}$ versus $\mathbf{q}$. The area under the curve is equal to $2 \pi^{2} Q$. The curves have very different shapes, but the areas under them are the same $\left(2 \pi^{2} \phi_{\mathrm{A}} \phi_{\mathrm{B}} V=5 \pi / 6\right)$. Figure 6 is a plot of $I(\mathbf{q}) \mathbf{q}^{4}$ versus $\mathbf{q}$. The limiting high $\mathbf{q}$ value in each case is $2 \pi S=1$. The phase sizes from eq 3 are $R_{\mathrm{A}}=10$ and $R_{\mathrm{B}}=5$ for each of the three distributions, as stated earlier.

\section{MONTE CARLO CALCULATIONS}

As a final test of the accuracy of the transformation method, Monte Carlo calculations have been made for the three model distributions. Random numbers $\left(N_{\mathrm{R}}\right)$ are generated in a rectangular distribution between 0 and 1. Equation 9 is integrated to give a cumulative probability, and eq 22 is solved with Newton's method to give a phase size $R_{\mathrm{R}}$ :

$$
\begin{aligned}
& \int_{R_{\mathrm{R}}}^{\infty} \sum_{i} w_{i} \exp \left(-R / R_{i}\right) / R_{i} d R \\
&=\sum_{i} w_{i} \exp \left(-R_{\mathrm{R}} / R_{i}\right)=N_{\mathrm{R}}
\end{aligned}
$$

After 10,000 alternating A and B phases are generated, correlations are calculated between the A-A combinations for values of the measuring rod up to 50 , and the process is repeated 100 times.

Figure 7 is a plot of the Monte Carlo calculated $\gamma(R)$ values along with the results from the Laplace calculations. Although the Monte Carlo calculations offer no advantages over the Laplace calculations in this example, they are a versatile way of calculating correlations in more complex systems. For example, if pores become filled with a contrast matching fluid through capillary condensation, the resulting correlation function and 


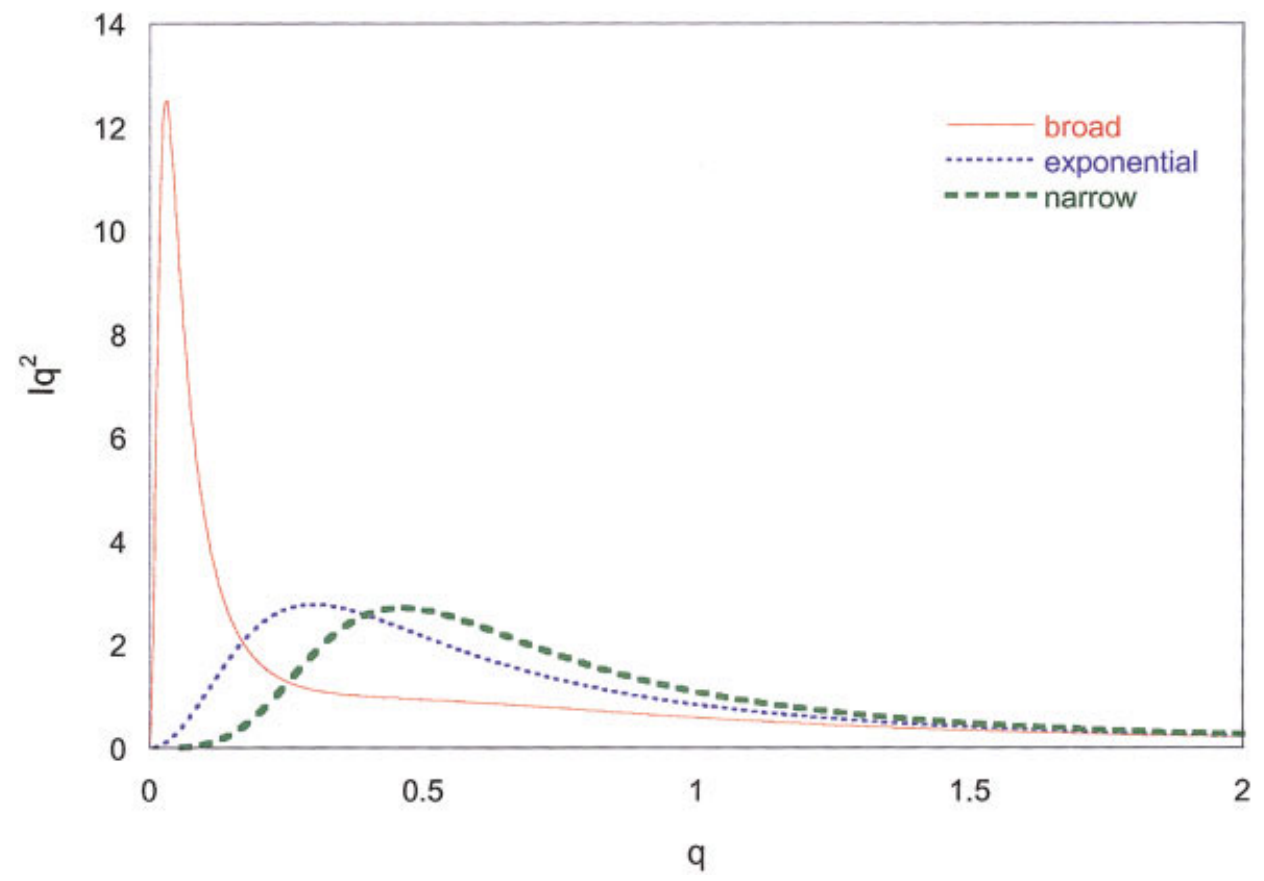

Figure 5. Kratky plots of the model functions described by eqs 13-15. The areas under the curves give the invariant. [Color figure can be viewed in the online issue, which is available at www.interscience.wiley.com]

hence small-angle scattering can be directly calculated through Monte Carlo methods when analytical methods are unavailable. Similarly, mul- tiphase morphologies or ones in which there are correlations between neighboring phases can be directly modeled. However, it is far more practical

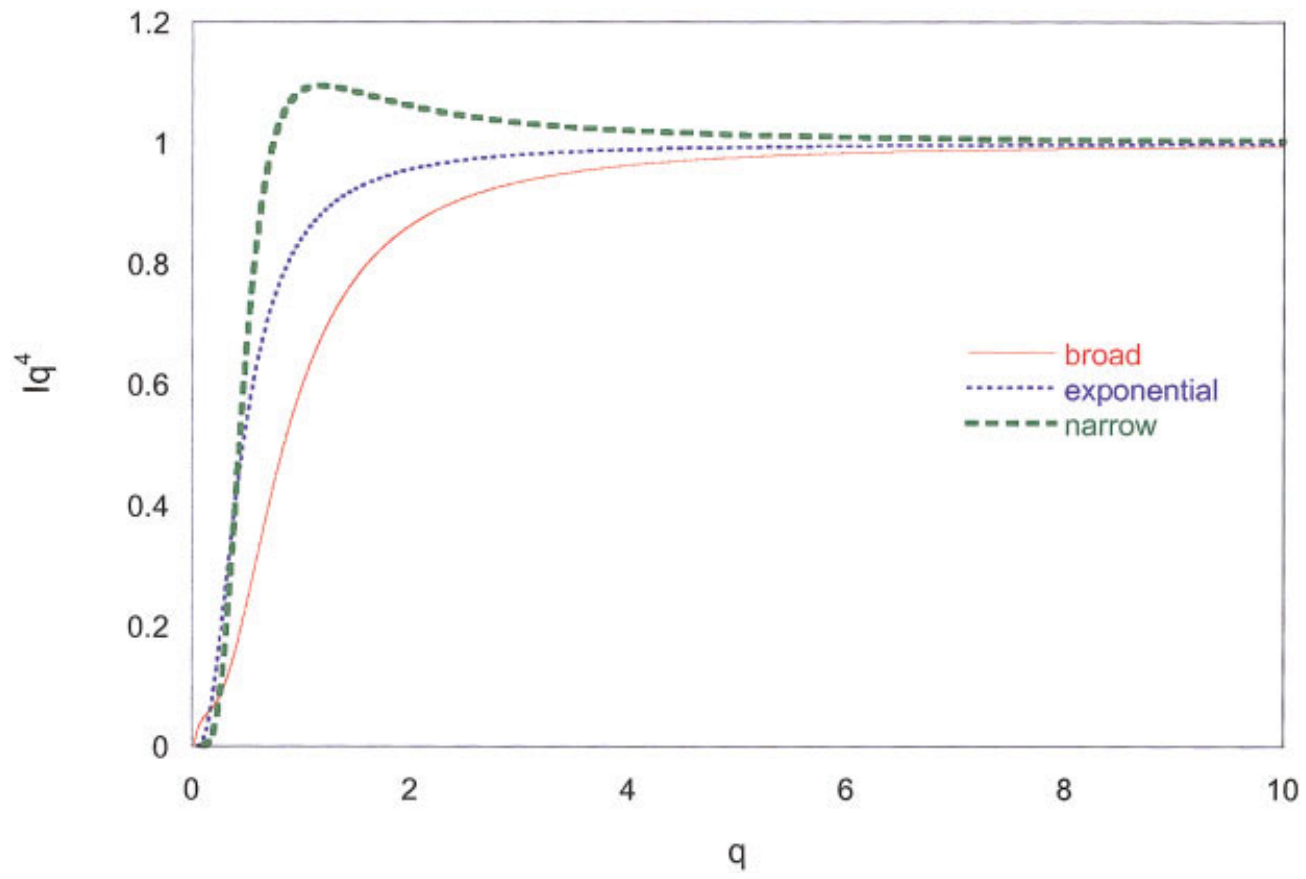

Figure 6. Porod plots of the model functions described by eqs 13-15. The high q plateau is the Porod limit. [Color figure can be viewed in the online issue, which is available at www.interscience.wiley.com] 


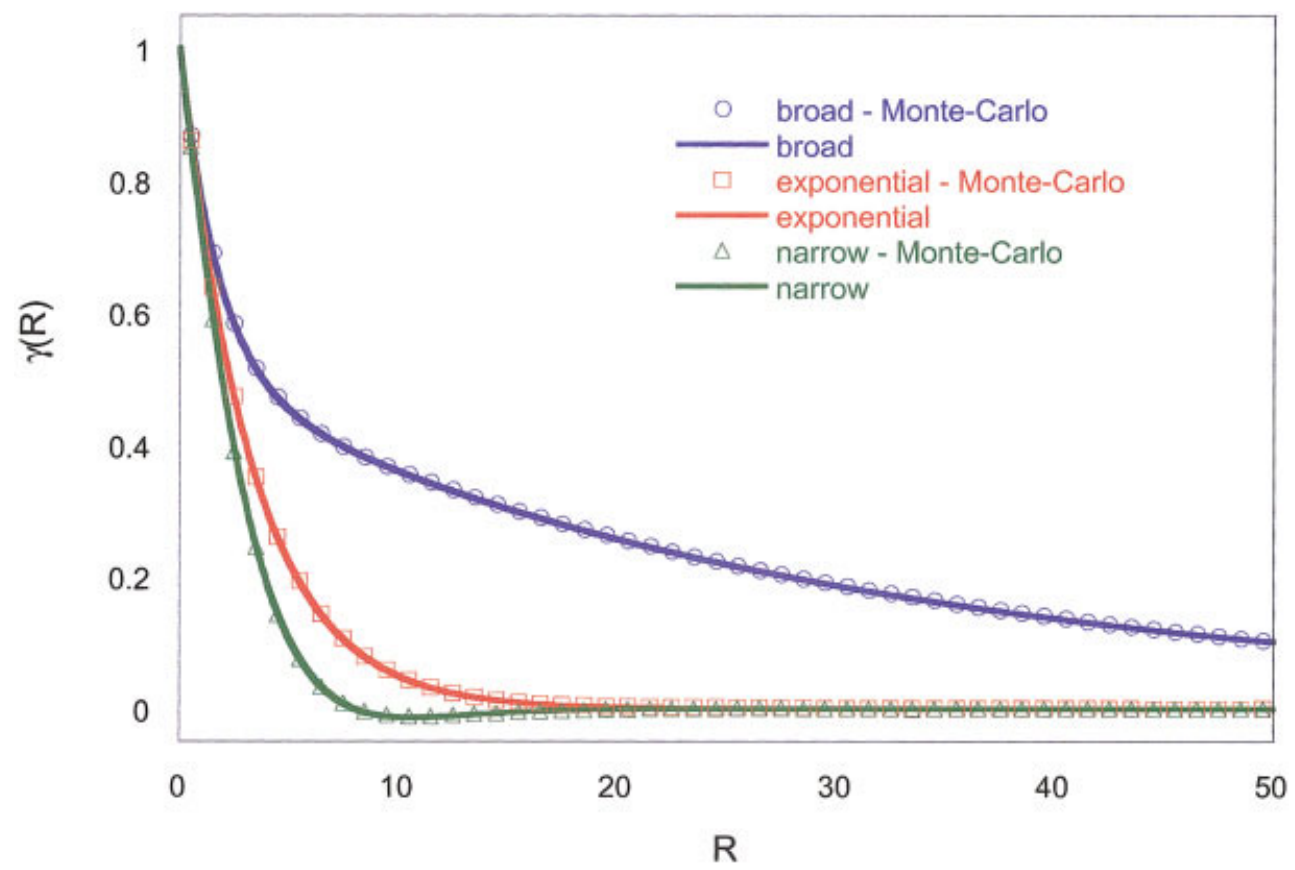

Figure 7. Correlation functions of the model distributions described by eqs 13-15 along with Monte Carlo results. [Color figure can be viewed in the online issue, which is available at www.interscience.wiley.com]

to fit morphologies that can be approximated by phase size distributions made up of a sum of exponential components with the Laplace transformation method.

\section{REVERSE TRANSFORMATIONS}

The Laplace method is a practical way of transforming any phase size distribution that can be fit by a sum of exponentials into the scattering intensity. The reverse procedure of calculating the phase size distribution from scattering data cannot be done because information is lost when structural information is transformed into the scattered intensity. Assumptions for the correlations between the distributions of the two phases need to be made, either thermodynamic or statistical. An example of a fitting procedure for smallangle neutron scattering (SANS) data involves calculating the equivalent distribution of the two phases; the two phases are assumed to have different average sizes but have the same distribution shape. This is the case when the $\mathrm{A}$ and $\mathrm{B}$ phases are described by the distribution of eq 9 with $w_{i}$ (phase $\mathrm{A}$ ) $=w_{i}$ (phase $\mathrm{B}$ ) and $R_{i}$ (phase $\mathrm{A}) / \phi_{\mathrm{A}}=R_{i}$ (phase B) $/ \phi_{\mathrm{B}}$. Although such an approximation does not distinguish the differences between the phases, it does provide an important measure of the polydispersity as a whole.

SANS measurements were conducted at the National Institute of Standards and Technology Center for Cold Neutron Research 8-m facility. The SANS experiments were carried out on a nanoporous thin film designed for spin-on, nanoporous, low- $k$ dielectric materials. The description of the SANS experiment and the data reduction protocols are reported elsewhere. ${ }^{2}$ The $I(\mathbf{q})$ data are reduced to an absolute intensity with secondary standards and appear in units of the scattered intensity per unit of volume. Films of this sort have been extensively analyzed by the Debye model. A single example is given of a random material that requires multiple exponential terms in eq 9 for the fitting of the SANS data.

Figure 8 shows the fit of the SANS data; two characteristic distances are assumed in eq 9 . The fits are made by nonlinear least squares, and the uncertainties are one standard deviation based on the goodness of the fit. The SANS uncertainties are not plotted, and one standard deviation is less than the symbol size. A fit is made of two exponential terms in eq 9 by a nonlinear-least-squares fit of the unweighted SANS intensity over a $\mathbf{q}$ range of 0.011-0.150 $\AA^{-1}$, with the uncertainties being one standard deviation based on the good- 


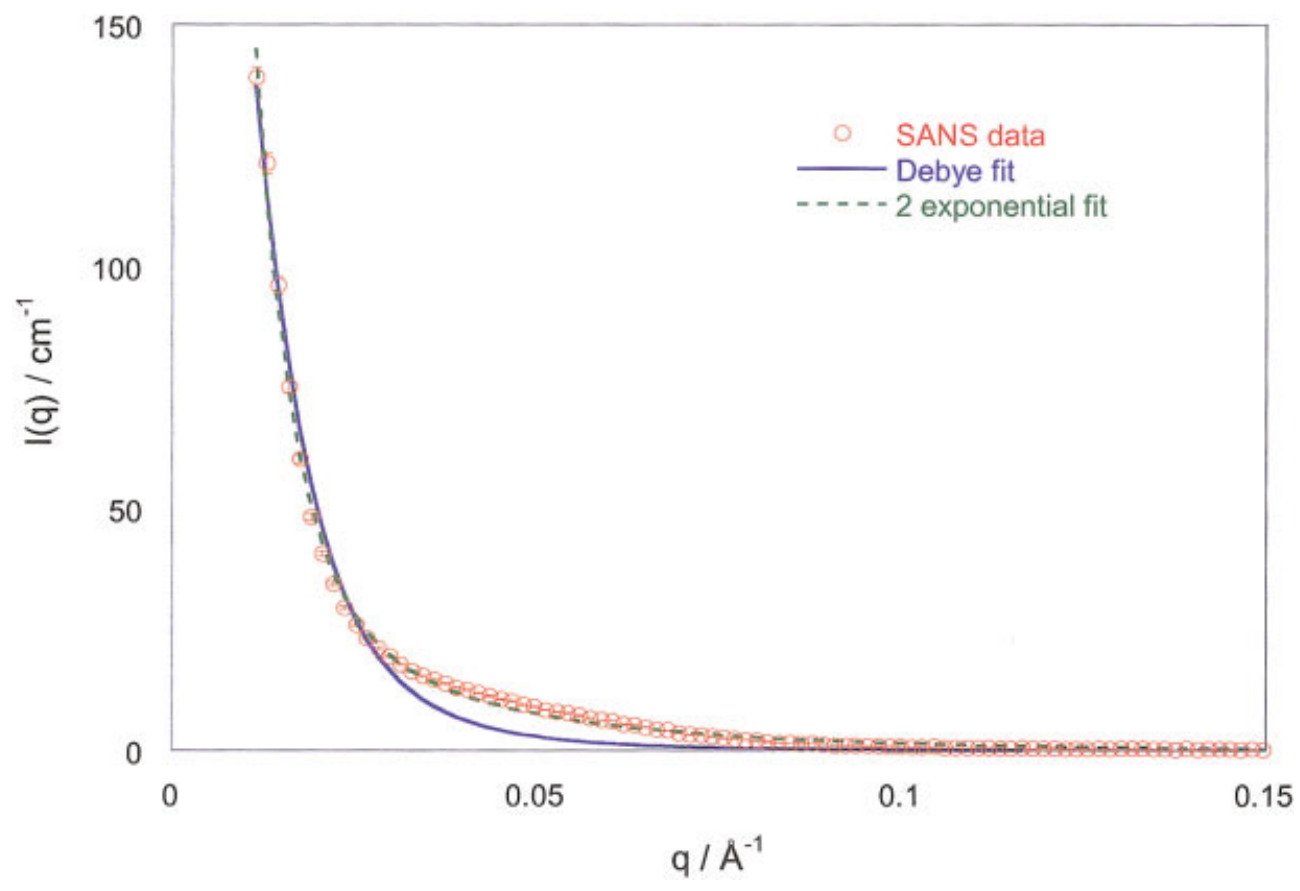

Figure 8. SANS data from a porous thin film, a fit with a two-exponent phase size distribution, and a Debye fit. [Color figure can be viewed in the online issue, which is available at www.interscience.wiley.com]

ness of the fit. The fit values are $R_{1}=16.2 \pm 0.1$ $\AA, R_{2}=81.0 \pm 0.3 \AA, w_{1}=0.962 \pm 0.031$, and $w_{2}$ $=0.038 \pm 0.001$. The fit is better over the whole $\mathbf{q}$ range than a single Debye fit. Many nanoporous, low- $k$ thin films have been successfully fit with a Debye function. ${ }^{2,4}$ The data analysis procedure

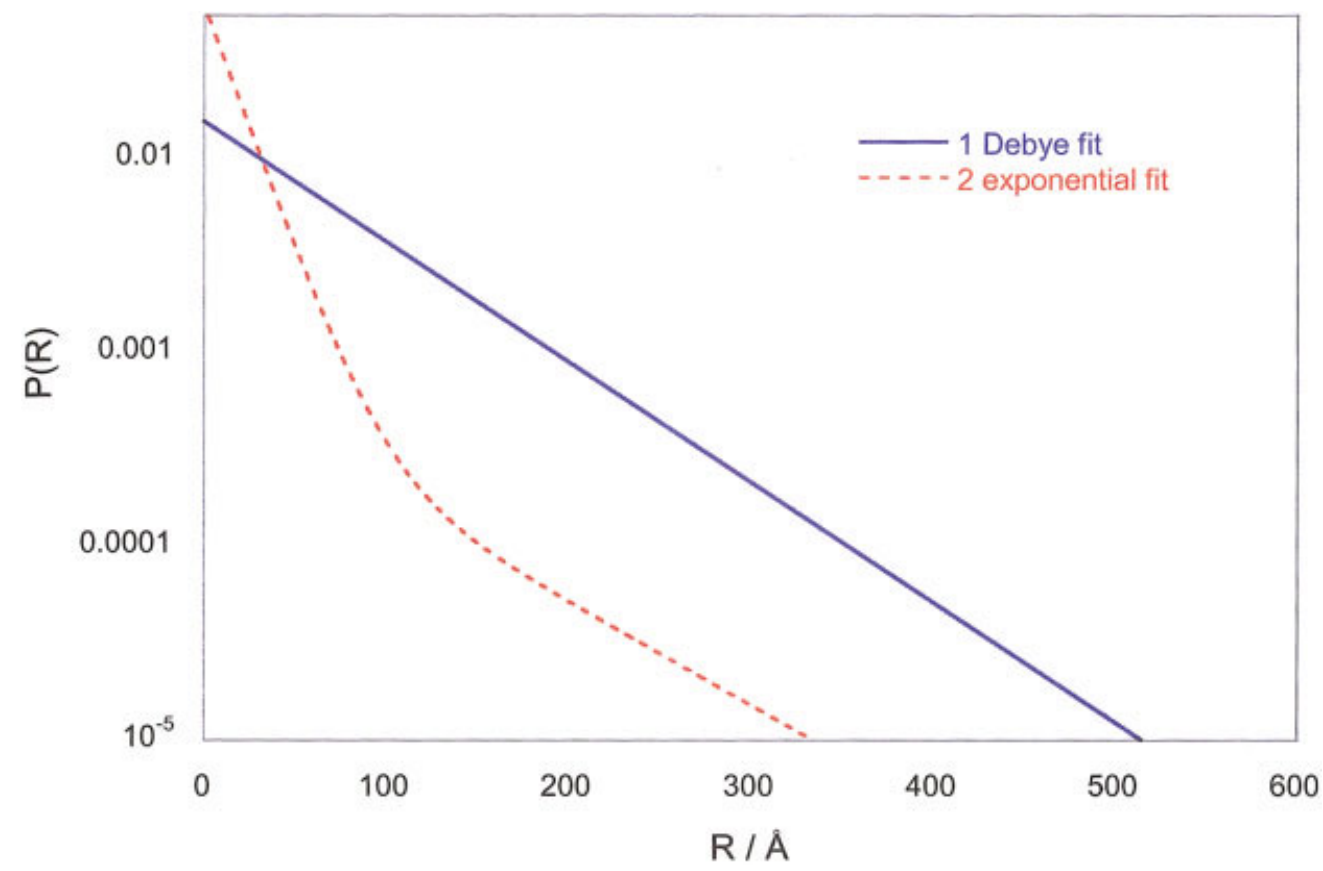

Figure 9. Phase size distributions of the two fits shown in Figure 8. [Color figure can be viewed in the online issue, which is available at www.interscience.wiley.com] 
calculates the invariant from the Debye functional form of the scattering and uses additional data from X-ray reflectivity to determine both the wall density and the volume fraction pores. This method cannot be used for morphologies such as that present in the SANS example of Figure 8. If a Debye equation is fit to the scattering, as shown in Figure 8, the invariant calculated from the fit is greatly underestimated. The Debye fit, which strongly weights the low $\mathbf{q}$ region, gives an average pore size of $70.0 \pm 0.4 \AA$, whereas the twoexponential fit gives $16.2 \pm 0.1 \AA$ A Figure 9 shows the calculated phase size distributions of the two types of fits. The Debye fit greatly overestimates the pore size and does not find the bimodal distribution.

\section{CONCLUSIONS}

A practical method of transforming phase size distributions of random phases into density correlation functions has been demonstrated. The computations are rapid and can produce density correlation functions and hence scattered intensities to any necessary degree of accuracy. Phase size distributions other than the single-exponential ones described by Debye et al. ${ }^{10}$ can be transformed into density correlations. Examples have been given of distributions that are broader than the single-exponential distribution (Debye) and narrower distributions that can lead to a peak in the scattering. Also, the Laplace transformation method uses information from the complete set of probability terms and avoids any errors introduced by a truncated series of the terms.

The transformation of the scattered intensity into model phase size distributions is possible with this method if assumptions are made of the relationship between the phases. An example of the transformation of small-angle scattering into a phase size distribution shows that for some systems, assuming Debye scattering produces significant discrepancies in the phase size distribution in comparison to fitting with multiple exponentials.

The author thanks International Sematech for the samples and for financial support and the Office for Microelectronics Programs at the National Institute of Standards and Technology for financial support. He also thanks Hae-Jeong Lee for helping with the small- angle neutron scattering data collection and Derek Ho and Charles Glinka NIST Center for Neutron Research for maintaining the 8-m small-angle neutron scattering equipment.

\section{REFERENCES AND NOTES}

1. Roe, R.-J. In Methods of X-Ray and Neutron Scattering in Polymer Science; Oxford University Press: New York, 2000; pp 134-209.

2. Bauer, B. J.; Lin, E. K.; Lee, H. J.; Wang, H.; Wu, W. L. J Electron Mater 2001, 30, 304-308.

3. Huang, E.; Toney, M. F.; Volksen, W.; Mecerreyes, D.; Brock, P.; Kim, H. C.; Hawker, C. J.; Hedrick, J. L.; Lee, V. Y.; Magbitang, T.; Miller, R. D.; Lurio, L. B. Appl Phys Lett 2002, 81, 2232-2234.

4. Wu, W. L.; Wallace, W. E.; Lin, E. K.; Lynn, G. W.; Glinka, C. J.; Ryan, E. T.; Ho, H. M. J Appl Phys 2000, 87, 1193-1200.

5. Belloni, L. In Neutron, X-Ray and Light Scattering: Introduction to an Investigative Tool for Colloidal and Polymeric Systems; Linder, P.; Zemb, T., Eds.; North Holland: New York, 1991; pp 135-155.

6. Griffith, W. L.; Triolo, R.; Compere, A. L. Phys Rev A 1986, 33, 2197-2200.

7. Griffith, W. L.; Triolo, R.; Compere, A. L. Phys Rev A 1987, 35, 2200-2206.

8. Pedersen, J. S. J Appl Crystallogr 1994, 27, 595608.

9. Porod, G. Kolloid-Z 1951, 124, 83.

10. Debye, P.; Anderson, H. R.; Brumberger, H. J Appl Phys 1957, 28, 679.

11. Berk, N. F. Phys Rev Lett 1987, 58, 2718-2721.

12. Quintanilla, J.; Reidy, R. F.; Gorman, B. P.; Mueller, D. W. J Appl Phys 2003, 93, 4584-4589.

13. Torquato, S.; Lu, B. Phys Rev E 1993, 47, 29502953.

14. Levitz, P.; Tchoubar, D. J Phys I 1992, 2, 771-790.

15. Mering, C.; Tchoubar, J. C R Acad Sci Paris 1966, 262, 1703.

16. Tchoubar, D.; Mering, J. J Appl Crystallogr 1969, 2, 128-138.

17. Tchoubar, D. In Neutron, X-Ray and Light Scattering: Introduction to an Investigative Tool for Colloidal and Polymeric Systems; Linder, P.; Zemb, T., Eds.; North Holland: New York, 1991; pp 157-174.

18. Smarsly, B.; Goltner, C.; Antonietti, M.; Ruland, W.; Hoinkis, E. J Phys Chem B 2001, 105, 831-840.

19. Smarsly, B.; Antonietti, M.; Wolff, T. J Chem Phys 2002, 116, 2618-2627.

20. Goltner, C. G.; Smarsly, B.; Berton, B.; Antonietti, M. Chem Mater 2001, 13, 1617-1624.

21. Smarsly, B.; Polarz, S.; Antonietti, M. J Phys Chem B 2001, 105, 10473-10483. 